\title{
Stress-adaptation among Family of Adolescent with Substance Misuse: Systematic Literature Review
}

\author{
Heru Subekti ${ }^{1 *}$ D, Ibrahim Rahmat ${ }^{2}$, Siswanto Wilopo ${ }^{3} \mathbb{D}$ \\ ${ }^{1}$ Doctorate Programme, Department of Mental Health and Community, School of Nursing, Faculty of Medicine, Public Health \\ and Nursing, Universitas Gadjah Mada, Yogyakarta, Indonesia; ${ }^{2}$ Department of Mental Health and Community, School of \\ Nursing, Faculty of Medicine, Public Health and Nursing, Universitas Gadjah Mada, Yogyakarta, Indonesia; ${ }^{3}$ Department of \\ Public Health, Faculty of Medicine, Public Health and Nursing, Universitas Gadjah Mada, Yogyakarta, Indonesia
}

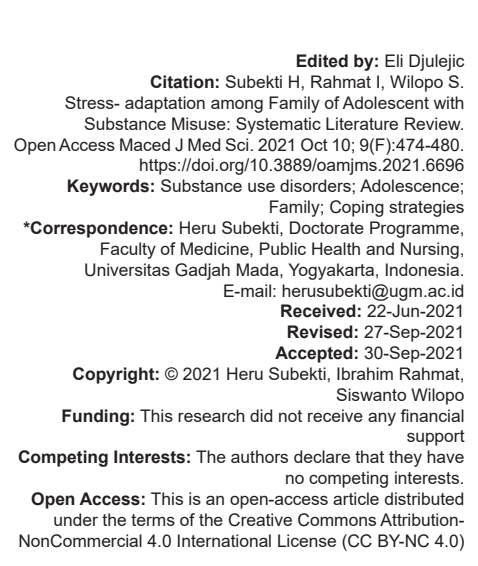

Introduction

Substance use disorders among teenagers have become a global issue [1]. It is a complex public health problem that is increasing worldwide [2]. Alcohol, cigarettes, and drugs are the most widely used substances, especially among adolescents. The World Health Organization (WHO) predicts that alcohol, cigarettes, and illegal drugs contributed $12.4 \%$ of the causes of death in 2000 [3]. The global mortality trends and burden of disease estimate ranks substance abuse as five out of the top six "youth killers" and have the fastest growth in causing global death [4].

Alcohol consumption is a factor that is often associated with an increased risk of experiencing mental health problems [5]. In addition, drug misuse and/ or abuse has been linked to mental health problems, which are most often associated with conduct disorder and attention deficit-hyperactivity disorder [6].

The impacts resulting from such problems include behavioral disorders and substance use disorder, which affect not only the sufferer but also the people involved with the person addicted, especially his/her family [7], [8]. It is estimated that any drug abuse problem will have a minimal impact on at least two other family members [9]. Drug abuse and alcohol consumption can cause stress, economic burden, family, and social dysfunction with routine disruption and the family may experience a heavy care burden [9], [10], [11], [12]. The disrupted family function can result in decreased relationship between nuclear family members including father, mother, and child relationships [12]. Low economic levels tend to have heavier burdens than high income families and people living in rural areas appear to have heavier burdens than those in urban areas [10]. Some studies found that families with high alcohol consumption show lower levels of closeness and life satisfaction than families without alcohol problems [13], [14]. Family members with alcohol use problems do not have adequate ways to deal with stress and face more stressful experiences compared to families with family members with schizophrenia problems. Furthermore, families often have obstacles in seeking health assistance. According 
to one study, the Australian National Narcotics Council reported that family members living with substance dependency have low awareness of finding sources of support, and families also have problems accessing services, and also have fears of social stigma [15]. Coping is defined as a person's ongoing cognitive and behavioral efforts to cope with certain external and/ or internal demands that are deemed to be taxing or surpassing the person's resources or abilities to cope [16]. This study aimed to assess coping and adjustment on the family of adolescent substance use disorders, using a systematic review. Identifying coping factors associated with adjustment or stress resilience can inform future research and health providers aiming to support the family health and well-being.

\section{Methods}

This systematic review of the literature used the Preferred Reporting Items for Systematic Reviews and Meta-Analysis methodology (Figure 1) [17]. The search was done in February 2019. The study was conducted through the following two online databases. Two online data-based MEDLINE (PubMed), EBSCO databases to select studies on coping involving the family of adolescents published in English from January 2000 to February 2019. The search terms were coping, family, caregivers, strategy, adaptation, family, behavior, adolescent, and substance use disorders in the following combinations:

\section{MEDLINE:}

((((((“"Parents”[Mesh]) OR “Fathers”[Mesh]) OR "Mothers"[Mesh])OR"Family"[Mesh]))AND((coping) OR "Adaptation, Psychological"[Mesh]))) AND (()((()“Underage Drinking"[Mesh]) OR “OpioidRelated Disorders"[Mesh]) OR drug user) OR "Marijuana Smoking"[Mesh])OR "Smoking"[Mesh]) OR "Alcohol Drinking"[Mesh]) OR "Substance-Related Disorders"[Mesh]).

\section{EBSCO:}

(Family or families or relatives or parents or siblings or caregiver) AND (family stress or family stressors) AND (family adaptation or family adjustment or family coping) AND (adolescents or teenagers or young adults or teen or youth or student or adolescence) AND (substance abuse or substance use or drug abuse or drug addiction or drug use) AND (adolescent substance abuse or teen drug abuse or adolescent drug use) AND (opioid use disorder or opioid related disorder or opioid dependence or opioid addiction or opioid misuse or opioid prescription misuse or opioid abuse or alcoholism or alcohol dependence or alcohol abuse or alcoholic or alcohol addiction or alcohol use disorder or smoking or tobacco or cigarette or nicotine).

\section{Study selection}

This study aimed to verify the experience of nuclear families in caring for family members of drug users. Drug users in this study are adolescents, while the nuclear family members in this study are father, mother, and siblings. All articles that explore the experiences of parents or siblings in dealing with adolescents with drug use issues were selected, while adolescent drug users with other health problems were not included in this study. Duplicates were removed and results were reviewed based on titles and abstracts. Full texts were retrieved for eligible studies and further reviewed for inclusion and exclusion criteria. Data were extracted from articles using a piloted data extraction form that included information about aims, design, sample, measures, and findings.

\section{Inclusion and exclusion criteria}

Inclusion criteria for quantitative studies were that they (a) reported experiences of parent or family; (b) investigated stress experience; (c) measured coping and adaptation and/or outcomes; and (d) include a quantitative and qualitative study on coping strategies of the family with adolescent substance use. Inclusion criteria for qualitative studies were that they (a) reported the experiences of parent or family; (b) investigated stress experience; and (c) discussed coping style/strategies. A control or comparison group was required for quantitative studies to reflect methodological quality but was not required for qualitative studies.

Studies were therefore excluded if they (a) were not the target population or topic (e.g., animal studies, informal caregivers, or did not investigate coping); (b) were a previous systematic review; and (c) were not written in English and a translation was not available. Two reviewers assessed articles against the criteria; the second reviewer conducted checking and confirmation, and disagreements were resolved through discussion. The synthesis process comprises (i) extracting themes and concepts from relevant research, (ii) organizing the outcomes of this extraction into key (primary) findings, (iii) grouping the findings into categories, and (iv) synthesizing the categories into a theme (adjusted with the conceptual framework that is prepared).

\section{Results}

Ten studies were found from the past 9 years (2000-2020). A summary table is provided for a quick reference (Table 1). Publication years ranged from 2000 to 2020. Qualitative data in all ten studies 
were collected through a semi-structured interview. Qualitative analysis was mostly thematic, alongside interpretive phenomenological analysis, and content analysis.

\section{Impact on the family}

Families often do not realize that their children have used drugs. Most parents assume that behavior changes that occur in their children are a natural stage, where they as adolescents often lie and speak disrespectfully. The family members sometimes felt devastated by the incidents of dishonesty and violent outbursts. They felt they failed as parents, and became very isolated from family and friends. This stigma happened because they were very ashamed of their children's behavior, but they still loved their sons and daughters [21]. The study conducted by McCann et al., on affected family members (AFM), examined family experiences related to the aggressive behavior of family members with drug use. The study was conducted with 31 AFM, 14 of who were parents. The study states that $70 \%$ of participants have experiences dealing with the use of problematic drug family members (problematic substance use [PSU]), especially those related to aggressive behavior. Aggressive behavior among drug users can occur dynamically and often unexpectedly, and simple problems can trigger aggressive behavior. This causes very irritating feelings for AFM and causes feelings of stress and emotional exhaustion [24].

According to other studies, family members of substance use disorders sometimes feel betrayed and have lost faith in themselves. Family interactions become defined by deception, stealing,

Table 1: Summary of selected studies

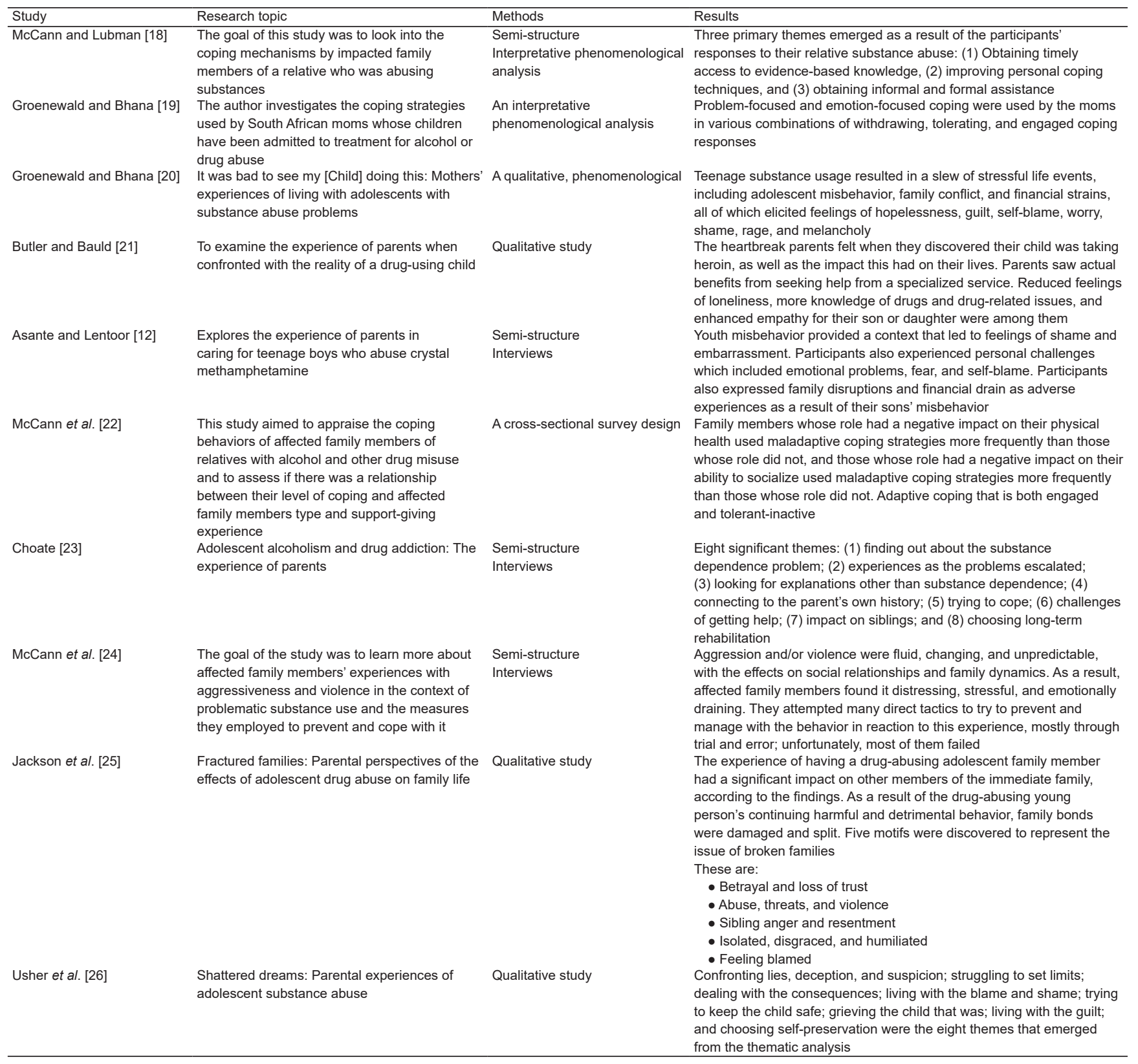


broken promises, and dishonesty, according to the participants. It was originally difficult for the participants to admit that their children had turned deceptive. They described themselves as being in denial, and unable to accept their situation [25].

Furthermore, research conducted by Choate et al., explored parents' experiences in dealing with adolescents who use drugs. It was explained that the family felt the negative impact from living with adolescents who use drugs which involved the occurrence of family function disorders such as loss of trust and family disharmony [12], [20], [23], [25].

Parents often feel their children do not respect them as parents, and they experience changes in their lives, including having severe emotional problems while experiencing panic and feeling very afraid of the condition. Financial problems are also experienced by parents, and they feel their money is drained away. In this condition of stress, the parents feel guilty about this condition, and they feel educating their children are done in vain [12]. The findings show that raising a child with a substance misuse problem is extremely difficult for parents.

The adolescent's substance use disorders can produce several stressful life events, such as adolescent misconduct (worry, anxiety, hopelessness, and shame), family conflict (anger and resentment), family disruption, and financial burdens, which are associated with different forms of emotional strain such as hopelessness, guilt, self-blame, worry, shame, anger, and signs of depression. In several qualitative studies, the parents spoke about the negative effects on their emotional or physical health.

Parents spoke about how they had been estranged from their families and friends. This was selfimposed for some, since they had made the explicit decision not to alert anyone about the condition. This was generally because they were embarrassed or ashamed of their child's drug usage and was concerned about what others would think or how they would react [12], [19], [20], [21], [24].

\section{Coping strategies among AFM}

\section{Emotional focus coping}

AFM feel overwhelmed by the stress they experience when treating family members with substance abuse [12], [18]. One study, conducted by Groenewald and Bhana, highlights the coping responses experienced by a mother when dealing with adolescents who are treated in the treatment of alcoholism. The study found a variety of complex coping approaches, which were discussed by the participants. Coping that arises is a matter of focus coping and emotional coping. Both coping strategies do not stand alone, but are combined with seeking, tolerating, and engaging in coping. The complexity of coping is also proven in various ways indicating that the same or similar coping approaches are applied by mothers. In addition, maternal coping responses are influenced by individual factors and the relationship between the "problem child" and mother [12], [19]. Emotional focus strategies are the coping responses most often performed on families [12], [19], [20], [21], [24]. Furthermore, Groenewald et al. discovered that many of the moms utilized both problem- and emotion-focused coping strategies, and that the same reaction can be both problem- and emotion-focused at times. One mother, for example, frequently used confrontational communication with her son or those she believed influenced her son's behavior. The careers' emotional emphasis, as evidenced by feelings of panic and uneasiness, arose predominantly as a result of the tremendous challenges linked with their children's behavior. The caregivers' unwillingness to seek aid or inability to talk to other people about their concerns causes panic attacks and uneasiness [12]. Family members who are affected by physical health tend to have maladaptive coping compared to family members who are not affected by physical health. Family members with disrupted social activities tend to have maladaptive treatment compared to family members whose social functions are not disrupted [22]. Research conducted by Jackson et al., involving 16 mothers and two fathers who have adolescents with drug use problems found that they experience emotional problems. As drug use continues, previously loving family relationships appear to show threats, harassment, and violence. Violence covers the whole spectrum from verbal, physical, and emotional violence to sexual abuse and suicide. These challenging and threatening circumstances evolve in such a way that in some cases other family members leave the family [25].

\section{Problem focus coping}

The study, conducted by McCann and Lubman, found that there are several strategies families use to deal with family members with drug abuse. There are three themes concluded in their study including finding sources of information, improving personal coping strategies, and finding sources of support. To improve their adaptive coping, participants stressed the significance of getting timely access to evidence-based information. Information from a variety of sources, particularly alcohol and other drug (AOD) services, was required. Information should focus on how AFMs may directly support relatives, treatment choices available, and how and where to get AOD services, according to AFMs. They also recognized the importance of being able to get knowledge to help them cope with the stress caused by their relative's substance abuse [18]. Research conducted by Usher et al. discussed the experience of parents in treating adolescents with drug use. Interviews were conducted with 18 parents living in rural and urban areas. This study found that there 


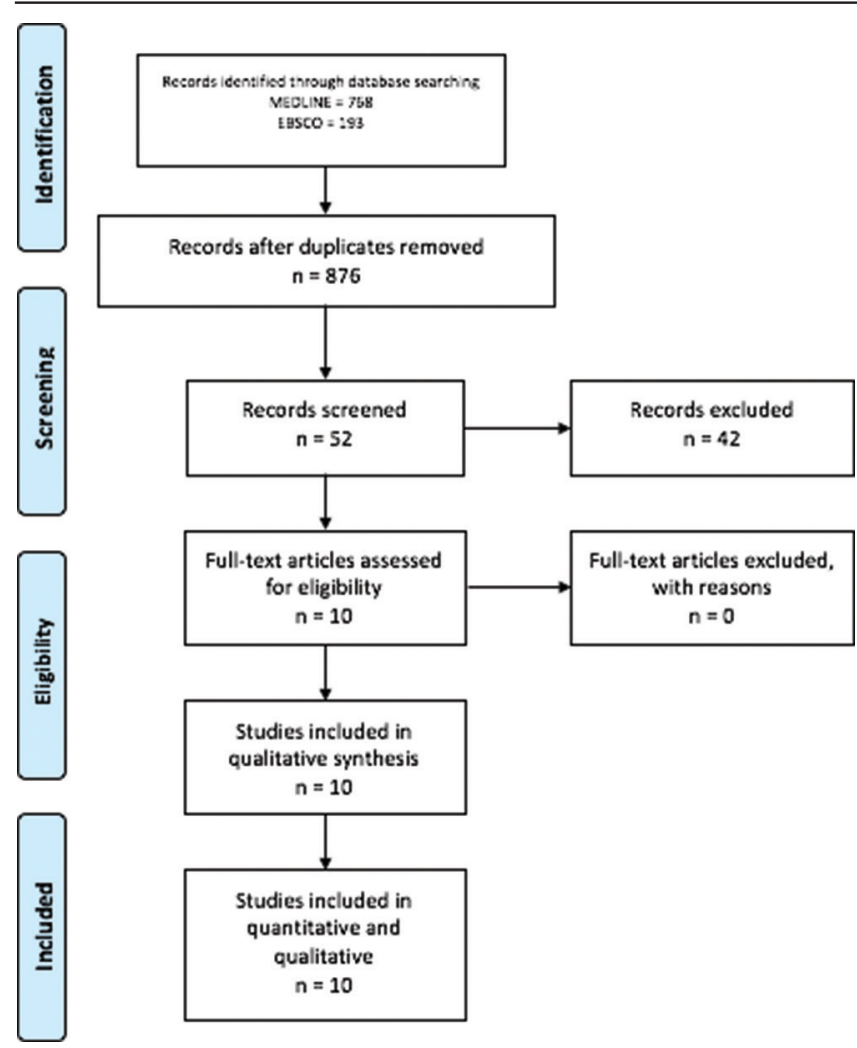

Figure 1: Flow Diagram based on Preferred Reporting Items for Systematic Reviews and Meta-Analysis guideline [17]

were eight themes raised. The findings revealed eight significant themes that shed light on the meanings of parenting a substance-addicted adolescent. These were: Confirming suspicions; struggling to set limits; dealing with the consequences; living with the blame and the shame; trying to keep the child safe; grieving the child that was; living with the guilt; and choosing self-preservation [26]. As parents attempted to regulate their adolescent's substance-abusing conduct, they attempted to place boundaries on the adolescent's behavior, particularly that which was seen to be related to the use of illegal substances. For most parents, it appeared to be a case of trial and error, in which they tried a variety of tactics in the hopes of finding one that worked. The individuals described an uphill battle in striving to successfully deal with substance use disorders behaviors [26]. The trial and error strategy made by families were also identified by McCann et al. indicating a limited number of options in responding to emotionally exhausting aggression and/or violence. Results showed mostly through trial and error, AFM identified several direct steps they used to prevent and overcome in this situation. However, most continue to struggle to deal with this behavior. They also highlighted additional indirect strategies, which if implemented, would enhance the existing strategies used to cope with aggression and/or violence. Most participants developed several strategies to try to prevent and cope with the behavior. Four main prevention and coping oriented strategies were developed: (1) Maintaining constant vigilance to avoid triggering aggression and/or violence, (2) curtailing social activities, disengaging from the person when PSU occurs, and (3) seeking help from law enforcement and judicial agencies [24]. Research conducted by Choate et al., which interviewed 31 adult caregivers of 21 adolescents, and produced eight important themes; (a) finding out about the substance dependence problem; (b) experiences as the problems escalated; (c) looking for explanations other than substance dependence; (d) connecting to the parent's own history; (e) trying to cope; (f) challenges of getting help; (g) impact on siblings; and (h) choosing longterm rehabilitation [23]. Parents typically reached out for formal help when they felt that the pressures arising from their youth's behaviors were now far beyond their abilities and capacities to cope. The majority of parents stated that they felt powerless and that nothing they tried appeared to work. Reaching out made sense after they broke through that barrier, and parents frequently discussed attempting almost anything to find a solution.

The majority of parents attempted to obtain formal help for their children through public health organizations or private resources [21], [23]. Some parents had contacted the group seeking basic information about medications and therapy choices for their children. Others, on the other hand, had gone out of their way to get help. This was frequently driven by a desire to learn why their child had gotten addicted to drugs [21].

\section{Engaged, tolerating, withdrawing}

Research conducted by Groenewald et al. identified the experiences of mothers about coping that arise when they find out their teenagers have substance abuse. The mothers were asked to reflect on how they coped with the distress they had experienced as a result of the adolescents' substance abuse behaviors. The analysis revealed that mothers' coping experiences were not homogenous. In addition, this study also revealed that a coping response in a mother in dealing with adolescent drug use can arise from more than one mechanism for coping. For example, some mothers will use a tolerant response and engage together. Mothers often feel that what teens do is caused by their divorce, and that this causes teenage drug use behavior. At the same time, some mothers were also alert and required ongoing drug testing which also showed the coping strategy that involved social pressures, in large part, causing her to take her son to an adolescent substance abuse treatment center [19]. Similarly, parents used a variety of coping strategies, including active coping and withdrawing. The engaged coping mechanism for these parents, where the majority were moms, included vigilance, talking to the teenager about his or her drug addiction, and establishing rules and sanctions to limit the adolescent's drug consumption. Among parents who distanced or retreated from the teenager, it was clear that they were withdrawing from the other. Similarly, research by Jackson et al. [25] showed that parents would disengage from their adolescents as a last and desperate attempt to cope. 
The engaged and tolerant-inactive coping subscales revealed the importance of the association between the effect on AFMs' physical health and socializing abilities in helping the relative with $A O D$ usage and subscale scores. AFMs who indicated that their support-giving role had a negative impact on their physical health and/or ability to socialize scored higher in both of these subscales than those who did not; as a result, the former group used these coping techniques more frequently than the latter [22].

\section{Discussion and Limitations}

We systematically reviewed the coping strategies used by the family of the adolescent with substance use disorders. Adolescent drug usage was perceived as a family crisis for these parents in the context of family life, and the findings from this study show the ongoing turbulence caused to families when young people develop substance abuse problems. Previous work by Butler and Bauld [21] found evidence that some parents began by denying that their children had problems related to drug abuse, but eventually parents sought help from a rehabilitation center to help them overcome the challenges they faced [27]. Parents in that study utilized active coping techniques including rigorous monitoring and limiting the child's independence, according to the researchers. For the development of interventions to support families who manage stress and the stress, they experience effectively requires a comprehensive understanding of the response of families in dealing with the problems they experience. The present research findings are consistent with the previous literature, which shows that family responses in dealing with substance abuse in adolescents are done in various ways [21], [25], [26]. Findings thus suggest that a simplified perspective that contrasts "tolerating," "engaging," and "withdrawing" coping strategies that are limited to one coping response is inadequate in terms of revealing the complexity of the family coping experience in handling various events. In another aspect, problems-focused and emotion-focused coping strategies cannot be separated, which contradict the theory put forward by Lazarus and Folkman [16]. Even though the coping mechanism is so complex, the emotional focus of coping becomes a response that is applied to almost every family. Problem focus coping such as family efforts to find sources of information and seek treatment services as a mechanism to be an adaptive coping strategy. Importantly, further research that investigates the experiences and roles of an affected family are necessary for both qualitative and quantitative methods. The results from this systematic review are limited to mostly western countries which have high profile of drug abuse and violence within dysfunctional families and are not easily generalizable to collective cultures such as those in Asia. Nevertheless, appropriate coping strategies can be identified as culturally sensitive and directed toward community support and empowerment in an effort to minimalize social stigma and heal families from the cycle of substance use disorder.

\section{Conclusion and Suggestion}

The research found that participants utilized a variety of coping techniques to deal with teenage substance use disorders issues. Problem-focused coping and emotional-focused coping are two of these coping techniques. Families, on the other hand, are more likely to utilize emotional focus coping as a coping method. Family engagement in finding information sources and seeking treatment services has proved to be more adaptable than other coping methods. These adjustments include a reduced influence on other family members and a greater capacity to solve the difficulties they are now confronted with. Mixed methods research that clearly define the sorts of coping techniques employed in various cultural and social status perspectives are still needed.

\section{References}

1. Kidd JD, Jackman KB, Wolff M, Veldhuis CB, Hughes TL. Risk and protective factors for substance use among sexual and gender minority youth: A scoping review. Curr Addict Rep. 2018;5(2):158-73. http://doi.org/10.1007/ s40429-018-0196-9

PMid:30393591

2. Smith JM, Estefan A. Families parenting adolescents with substance abuse: Recovering the mother's voice: A narrative literature review. J Fam Nurs. 2014;20(4):415-41. http://doi. org/10.1177/1074840714554397

PMid:25342471

3. World Health Organization. Management of Substance Abuse Geneva: World Health Organization; 2019. Available from: http://www.who.int/substance_abuse/en [Last accessed on 2019 Feb 11].

4. Mathers CD, Loncar D. Projections of global mortality and burden of disease from 2002 to 2030. PLoS Med. 2006;3(11):e442. http://doi.org/10.1371/journal.pmed.0030442

PMid:17132052

5. Rodgers B, Korten AE, Jorm AF, Christensen H, Henderson S, Jacomb PA. Risk factors for depression and anxiety in abstainers, moderate drinkers, and heavy drinkers. Addict Abingdon Engl. 2000;95(12):1833-45. http://doi. org/10.1046/j.1360-0443.2000.9512183312.x

PMid:11177498

6. Skogen JC, Sivertsen B, LundervoldAJ, StormarkKM, Jakobsen R, Hysing M. Alcohol and drug use among adolescents: And the co-occurrence of mental health problems. Ung @ hordaland, a 
population-based study. BMJ Open. 2014;4(9):e005357. http:// doi.org/10.1136/bmjopen-2014-005357

PMid:25245403

7. Kuhn ES, Laird RD. Family support programs and adolescent mental health: Review of evidence. Adolesc Health Med Ther. 2014;5:127-42. http://doi.org/10.2147/AHMT.S48057 PMid:25177156

8. Crowe A, Lyness KP. Family functioning, coping, and distress in families with serious mental illness. Fam J. 2013;22(2):186-97.

9. Copello A, Orford J, Vellem RA. Methods for reducing alcohol and drug related family harm in non-specialist settings. J Ment Health. 2000;9(3):329-43.

10. Mattoo SK, Nebhinani N, Kumar BN, Basu D, Kulhara P. Family burden with substance dependence: A study from India. Indian J Med Res. 2013;137(4):704-11. PMid:23703337

11. Orford J, Velleman R, Copello A, Templeton L, Ibanga A. The experiences of affected family members: A summary of two decades of qualitative research. 2010;17:44.

12. Asante $\mathrm{KO}$, Lentoor AG. Use of crystal methamphetamine among male adolescents in Cape Town, South Africa: Caregivers' experiences. Subst Abuse Treat Prev Policy Lond. 2017;12(1):18. http://doi.org/10.1186/s13011-017-0102-9 PMid:28347349

13. Park GH, Choi YJ. Family stress and coping from hospitalization of clients with severe alcohol use disorder in Korea. J Addict Nurs. 2017;28(1):4-10. http://doi.org/10.1097/ JAN.0000000000000154 PMid:28252506

14. Lee KM, Manning V, Teoh HC, Winslow M, LeeA, Subramaniam M, et al. Stress-coping morbidity among family members of addiction patients in Singapore. Drug Alcohol Rev. 2011;30(4):441-7. http://doi.org/10.1111/j.1465-3362.2011.00301.x PMid:21726307

15. Moriarty H, Stubbe M, Bradford S, Tapper S, Lim BT. Exploring resilience in families living with addiction. J Prim Health Care. 2011;3(3):210-7. PMid:21892423

16. Richard S. Lazarus. Stress, Appraisal, and Coping. Berlin, Heidelberg: Springer Publishing Company; 1984. p. 460.

17. Moher D, Liberati A, Tetzlaff J, Altman DG. Preferred reporting items for systematic reviews and meta-analyses: The PRISMA statement. BMJ. 2009;339:b2535.
18. McCann TV, Lubman DI. Adaptive coping strategies of affected family members of a relative with substance misuse: A qualitative study. J Adv Nurs. 2018;74(1):100-9. http://doi. org/10.1111/jan. 13405 PMid:28771795

19. Groenewald C, Bhana A. Mothers' experiences of coping with adolescent substance abuse: A phenomenological inquiry. Contemp Nurse. 2017;53(4):421-35. http://doi.org/10.1080/103 76178.2017.1361854 PMid:28760077

20. Groenewald C, Bhana A. "It was bad to see my [child] doing this": Mothers' experiences of living with adolescents with substance abuse problems. Int J Ment Health Addict. 2016;14(5):646-61.

21. Butler R, Bauld L. The parents' experience: Coping with drug use in the family. Drugs Educ Prev Policy. 2005;12(1):35-45.

22. McCann TV, Stephenson J, Lubman DI. Affected family member coping with a relative with alcohol and/or other drug misuse: A cross-sectional survey questionnaire. Int J Ment Health Nurs. 2018;28(3):687-96.

23. Choate PW. Adolescent alcoholism and drug addiction: The experience of parents. Behav Sci Basel Switz. 2015;5(4):461-76. http://doi.org/10.3390/bs5040461 PMid:26529024

24. McCann TV, Lubman DI, Boardman G, Flood M. Affected family members' experience of, and coping with, aggression and violence within the context of problematic substance use: A qualitative study. BMC Psychiatry. 2017;17(1):209. http://doi. org/10.1186/s12888-017-1374-3 PMid:28578666

25. Jackson D, Usher K, O'Brien L. Fractured families: Parental perspectives of the effects of adolescent drug abuse on family life. Contemp Nurse. 2006;23(2):321-30. http://doi.org/10.5555/ conu.2006.23.2.321 PMid:17343535

26. Usher K, Jackson D, O'Brien L. Shattered dreams: Parental experiences of adolescent substance abuse. Int J Ment Health Nurs. 2007;16(6):422-30. http://doi. org/10.1111/j.1447-0349.2007.00497.x PMid:17995513

27. Jackson D, Mannix J. Then suddenly he went right off the rails: Mothers' stories of adolescent cannabis use. Contemp Nurse. 2003;14(2):169-79. http://doi.org/10.5172/ conu.14.2.169

PMid:12785607 